\title{
RATIONAL CONIC FIBRATIONS OF SECTIONAL GENUS TWO
}

\author{
ANTONIO LANTERI, RAQUEL MALLAVIBARRENA
}

\begin{abstract}
Polarized rational surfaces $(X, \mathcal{L})$ of sectional genus two ruled in conics are studied. When they are not minimal, they are described as the blow-up of $\mathbb{F}_{1}$ at some points lying on distinct fibers. Ampleness and very ampleness of $\mathcal{L}$ are studied in terms of their location. When $\mathcal{L}$ is very ample and there is a line contained in $X$ and transverse to the fibers, the conic fibrations $(X, \mathcal{L})$ are classified and a related property concerned with the inflectional locus is discussed.
\end{abstract}

2010 Mathematics Subject Classification: Primary: 14C20, 14J26; Secondary: 14D06, 14N05; 51N35.

Keywords: Rational conic fibration; ample divisor; sectional genus; inflection point.

\section{INTRODUCTION}

In the study of osculation for projective varieties there is a large literature concerning scrolls over a curve. The investigation of quadric fibrations over a curve, from the same point of view, was started in [7]. In particular, the special case of dimension two, namely surfaces fibered in conics over a curve, is in itself interesting in some respects: for instance, though the expected maximal dimension of the osculating spaces does not distinguish them from a general surface, the special structure they have implies that the curve cut out by any osculating hyperplane is reducible, containing a fiber. In [6] we studied the osculatory behavior of smooth surfaces fibered in conics over a curve, and, as an application, we described the inflectional loci of a special class of such surfaces with sectional genus $g=2$, that we called Castelnuovo surfaces. This study oriented our interest towards a better understanding of how these surfaces are framed in the more general context of the rational conic fibrations polarized by an ample line bundle of sectional genus two. From this perspective, first we have to note that while in the context of very ample line bundles rational conic fibrations with $g=2$ coincide with rational surfaces with $g=2$, this is no longer true in the wider setting of ample line bundles. Actually, in Fujita's classification of polarized surfaces with $g=2$ [3. Theorem 15.2, pp. 122-123], they simply correspond to cases $6_{0}$ ) $-6_{2}$ ), up to blow-ups (see case 0) there), but other rational surfaces appear (cases $7), 8)$ ) besides conic fibrations. 
So, let $(X, \mathcal{L})$ be a polarized rational surface of sectional genus two ruled in conics. If $X$ is minimal, then $X \cong \mathbb{F}_{e}$, a Segre-Hirzebruch surface of invariant $e \geq 0$, and the fact that $\mathcal{L}$ is an ample line bundle of genus two immediately leads to determine its expression in terms of the generators of $\operatorname{Pic}(X)$ and to get the bound $e \leq 2$. If $X$ is not minimal, by means of elementary transformations, we can describe $X$ via a birational morphism $\eta: X \rightarrow \mathbb{F}_{1}$ as the blow-up of $\mathbb{F}_{1}$ at points $p_{1}, \ldots, p_{\mu}$, lying on distinct fibers, and then

$$
\mathcal{L}=\eta^{*} \mathcal{L}_{0}-\sum_{i=1}^{\mu} e_{i},
$$

where $\mathcal{L}_{0} \in \operatorname{Pic}\left(\mathbb{F}_{1}\right)$ is an ample line bundle of genus two and $e_{i}$ is the exceptional curve corresponding to $p_{i}$ for $i=1, \ldots, \mu$. Moreover, $\mu \leq 11$. Conversely, the line bundle $\mathcal{L}$ defined by (1) in this situation is ample provided that $p_{1}, \ldots, p_{\mu}$ are general enough, according to Yokoyama [12]. So, as a first thing we explore the precise conditions that the points $p_{1}, \ldots, p_{\mu}$ have to satisfy in order to ensure the ampleness of $\mathcal{L}$, and we make them explicit. They are expressed in the first part of Theorem 10. However, doing the same to ensure the very ampleness of $\mathcal{L}$, in which case, necessarily, $\mu \leq 7$, looks more delicate. Nevertheless, we succeed to do it thanks to Proposition 5 (2), Proposition 7 , and the characterization of the nefness and bigness of the anticanonical bundle we obtain by translating Demazure's almost general position condition for points in $\mathbb{P}^{2}[2]$ in terms of $\mathbb{F}_{1}$. This is summarized in the last part of Theorem 10.

In connection with this problem, one could refer to the paper by E. Bese [1, who studied, more generally, the spannedness and the very ampleness of line bundles of type (11), when $X$ is obtained by blowing-up any SegreHirzebruch surface $\mathbb{F}_{e}$ and $\mathcal{L}_{0}$ is any ample line bundle on it. However, some results of Bese specialized to the situation under consideration do not coincide with ours, unfortunately (see Remark 5 for details). Actually, according to [1, Theorem 4.2], it seems that if $\mathcal{L}_{0}$ has bidegree $(2,4)$, seven points $p_{i}$ 's could lie on a general curve of $\mathbb{F}_{1}$ of bidegree $(2,2)$ without affecting the very ampleness of $\mathcal{L}$. But this is not true in view of Proposition 7 and Remark 4. Moreover, the last part of Theorem 10 says that this is essentially the key difference. In fact further slight discrepancies simply derive from the fact that while the conditions we obtain characterize the very ampleness of $\mathcal{L}$, [1] only provides sufficient conditions for that.

In the final Section we provide the complete list of conic fibrations with $g=2$, polarized by a very ample line bundle, containing a line transverse to the fibers (Theorem 12). If $\mu \leq 6$ any such line, as well as those constituting the irreducible components of the reducible fibers, is contained in the inflectional locus of $X$ embedded in $\mathbb{P}^{N}$ by $|\mathcal{L}|$. This is not true however when $\mu=7$, and in this case we make explicit the condition ensuring that such a line is not contained in the inflectional locus (Proposition 13). 


\section{BACKGROUND}

All surfaces considered in this paper are smooth and defined over the complex field. We use the standard notation and terminology from algebraic geometry. In particular, we recall that a polarized surface is a pair $(X, \mathcal{L})$ consisting of a surface $X$ and an ample line bundle $\mathcal{L}$ on $X$. The degree and the sectional genus of $(X, \mathcal{L})$ are defined by $d(X, \mathcal{L}):=\mathcal{L}^{2}$ and $g(X, \mathcal{L}):=$ $1+\frac{1}{2}\left(K_{X}+\mathcal{L}\right) \cdot \mathcal{L}$, respectively. We say that a line bundle is spanned to mean that the corresponding invertible sheaf is generated by global sections. For any integer $e \geq 0$, we denote by $\mathbb{F}_{e}$ the Segre-Hirzebruch surface of invariant $e$, i.e. $\mathbb{F}_{e}=\mathbb{P}(\mathcal{E})$, where $\mathcal{E}=\mathcal{O}_{\mathbb{P}^{1}} \oplus \mathcal{O}_{\mathbb{P}^{1}}(-e)$. By $s$ and $f$ we denote the $(a$, if $e=0)$ tautological section of minimal self-intersection $s^{2}=-e$, and a fiber, respectively. We recall that the classes of $s$ and $f$ generate the Picard group of $\mathbb{F}_{e}$, hence, for any line bundle $\mathcal{L}$ on $\mathbb{F}_{e}$ we can write $\mathcal{L}=[\alpha s+\beta f]$ for some integers $\alpha, \beta$. According to [4, Corollary 2.18, p. 380], $\mathcal{L}$ is ample, if and only if it is very ample, if and only if $\alpha>0$ and $\beta>\alpha e$. For further properties we refer to [4, Ch. V, Sec. 2]. In particular, when $\alpha=2$ (and $\beta>2 e)$, the polarized surface $\left(\mathbb{F}_{e}, \mathcal{L}\right)$ is a conic bundle. In this case, by the projection formula we have $h^{0}(\mathcal{L})=h^{0}\left(\pi_{*} \mathcal{L}\right)=h^{0}\left(S^{2} \mathcal{E} \otimes \mathcal{O}_{\mathbb{P}^{1}}(\beta)\right)$, where $\pi: \mathbb{F}_{e} \rightarrow \mathbb{P}^{1}$ is the bundle projection and $S^{2}$ stands for the second symmetric power. Note that

$$
S^{2} \mathcal{E} \otimes \mathcal{O}_{\mathbb{P}^{1}}(\beta)=\mathcal{O}_{\mathbb{P}^{1}}(\beta) \oplus \mathcal{O}_{\mathbb{P}^{1}}(\beta-e) \oplus \mathcal{O}_{\mathbb{P}^{1}}(\beta-2 e) .
$$

Moreover, all summands have positive degree, since $\beta>2 e$. Therefore,

$$
h^{0}(\mathcal{L})=3(\beta-e+1) .
$$

It is useful to recall that $\mathbb{F}_{1}$ is isomorphic to the projective plane $\mathbb{P}^{2}$ blownup at a point, say $q_{0}$. If $\tau: \mathbb{F}_{1} \rightarrow \mathbb{P}^{2}$ stands for this blow-up, then the tautological section $s$ is just the exceptional curve $\tau^{-1}\left(q_{0}\right)$. It follows that $\tau^{*} \mathcal{O}_{\mathbb{P}^{2}}(1)=[s+f]$, and then the general element in the linear system $|s+\beta f|$ is the proper transform via $\tau$ of an irreducible plane curve of degree $\beta$ having a singular point of multiplicity $\beta-1$ at $q_{0}$.

The following fact will be used often.

Remark 1. Let $r$ be any positive integer. On $\mathbb{F}_{1}$, we have $s \cap \gamma=\emptyset$ for any irreducible curve $\gamma \in|r(s+f)|$. This simply follows from the fact that $s \cdot(s+f)=0$.

\section{Rational CONiC Fibrations in the SETting OF POLARIZED SURFACES}

Let $X$ be a smooth projective surface and let $\mathcal{L}$ be any line bundle on $X$. We say that $(X, \mathcal{L})$ is a pre-conic fibration if $X$ is endowed with a fibration $\pi: X \rightarrow B$ over a smooth curve $B$ (i.e. $\pi$ is a morphism with connected fibers) such that the following conditions hold:

$$
\left(F, \mathcal{L}_{F}\right)=\left(\mathbb{P}^{1}, \mathcal{O}_{\mathbb{P}^{1}}(2)\right) \text { for any general fiber } F \text { of } \pi
$$


moreover, singular fibers, if any, are reducible and each consists of

$$
\text { two (-1)-curves } \ell, \ell^{\prime} \text { with } \ell \cdot \ell^{\prime}=\mathcal{L} \cdot \ell=\mathcal{L} \cdot \ell^{\prime}=1 \text {. }
$$

Note that if $\mathcal{L}$ is ample then (44) is a consequence of (3). In this case we say that the polarized surface $(X, \mathcal{L})$ is a conic fibration. We will use the expressions pre-conic bundle and conic bundle to mean that $X$ has no singular fibers, i.e., that it is a $\mathbb{P}^{1}$-bundle. If $B=\mathbb{P}^{1}$, then $X$ is a rational surface and we say that $(X, \mathcal{L})$ is a rational pre-conic fibration. For such a pair, let $g$ be the (virtual) arithmetic genus of $\mathcal{L}$. First of all we point out the following fact. Let $F$ be a fiber of $\pi$. Since $F^{2}=0$ we have $K_{X} \cdot F=-2$ by the genus formula. On the other hand, $\mathcal{L} \cdot F=2$. So $\left(K_{X}+\mathcal{L}\right) \cdot F=0$, and $\left(K_{X}+\mathcal{L}\right) \cdot \ell=0$ for any irreducible component $\ell$ of a reducible fiber, by (4), hence $K_{X}+\mathcal{L}=\pi^{*} \mathcal{O}_{\mathbb{P}^{1}}(\alpha)$ for some integer $\alpha$. In particular, $\left(K_{X}+\mathcal{L}\right)^{2}=0$; furthermore, $2 g-2=\mathcal{L} \cdot\left(K_{X}+\mathcal{L}\right)=\mathcal{L} \cdot \alpha F=2 \alpha$, which gives

$$
\mathcal{L}=-K_{X}+(g-1) F .
$$

In particular, we get $\mathcal{L}^{2}=K_{X}^{2}+4(g-1)$. On the other hand, let $\mu$ be number of singular fibers of $\pi: X \rightarrow \mathbb{P}^{1}$. By contracting an irreducible component of every singular fiber of $\pi$ we get a birational morphism from $X$ to an $\mathbb{F}_{e}$, factoring through $\mu$ blowing-ups; hence $K_{X}^{2}=8-\mu$, and then

$$
\mathcal{L}^{2}=4(g+1)-\mu \text {. }
$$

This applies in particular, when $\mathcal{L}$ is ample, to polarized rational conic fibrations, that we simply call rational conic fibrations when there is no ambiguity. In this case $g=g(X, \mathcal{L})$ is the sectional genus and $d=d(X, \mathcal{L})$ is the degree of $(X, \mathcal{L})$. Then $d>0$, and $g \geq 0$. Since $X$ has Picard number $\rho \geq 2$, we note that $g=0$ if and only if the rational conic fibration $(X, \mathcal{L})$ has at the same time the structure of a rational scroll [8, Corollary 2.3], and this can happen only for $\left(\mathbb{P}^{1} \times \mathbb{P}^{1}, \mathcal{O}_{\mathbb{P}^{1} \times \mathbb{P}^{1}}(1,2)\right), \pi$ being the projection onto the first factor. We can thus assume that $g \geq 1$. Moreover, we note that rational conic fibrations with $g=1$ are simply the pairs $\left(X,-K_{X}\right)$, where $X$ is a del Pezzo surface distinct from $\mathbb{P}^{2}$. This explains the interest for case $g=2$.

Proposition 1. Let $(X, \mathcal{L})$ be a rational conic fibration. Then $\mu \leq 4 g+3$. Moreover, $d \leq 4(g+1)$, with equality if and only if

$$
(X, \mathcal{L})=\left(\mathbb{F}_{e},[2 s+(g+e+1) f]\right)
$$

for some integer $0 \leq e \leq g$.

Proof. The first inequality is obvious in view of (6), since $d \geq 1, \mathcal{L}$ being ample. The second inequality is simply due to the fact that $\mu \geq 0$ and the characterization of the equality follows from the properties of the SegreHirzebruch surfaces. Once we know that $X=\mathbb{F}_{e}$ for some $e \geq 0$, the expression of $\mathcal{L}$ follows from the genus formula. Finally, the fact that $s \cdot \mathcal{L}=$ $-e+g+1>0$, due to the ampleness of $\mathcal{L}$, implies that $e \leq g$. 
Remark 2. Clearly, the upper bound for $\mu$ provided by Proposition 1 can be improved if the ample line bundle $\mathcal{L}$ satisfies some additional requirement. Actually, if $\mathcal{L}$ is ample and spanned, then necessarily $\mathcal{L}^{2} \geq 2$ and equality holds if and only if either $(X, \mathcal{L})=\left(\mathbb{P}^{1} \times \mathbb{P}^{1}, \mathcal{O}_{\mathbb{P}^{1} \times \mathbb{P}^{1}}(1,1)\right)$, which however is not a conic fibration, or there exists a finite morphism $\varphi: X \rightarrow \mathbb{P}^{2}$ of degree 2 such that $\mathcal{L}=\varphi^{*} \mathcal{O}_{\mathbb{P}^{2}}(1)$. Let $2 b$ be the degree of the branch divisor; then $K_{X}=\varphi^{*} \mathcal{O}_{\mathbb{P}^{2}}(-3+b)=(b-3) \mathcal{L}$ by the ramification formula and so the condition $\left(K_{X}+\mathcal{L}\right)^{2}=0$ implies $b=2$, i.e. $\varphi$ is the del Pezzo double plane, in which case $g=1$. As a consequence, if $\mathcal{L}$ is ample and spanned, then $\mu \leq 6$ if $g=1$, while $\mu \leq 4 g+1$ if $g \geq 2$. We have a stronger improvement assuming that $\mathcal{L}$ is very ample. Actually, by restricting the fibration morphism $\pi: X \rightarrow \mathbb{P}^{1}$ to any smooth curve $C \in|\mathcal{L}|$ we get a morphism $\left.\pi\right|_{C}: C \rightarrow \mathbb{P}^{1}$ of degree 2 ; so either $g=1$ or $C$ is hyperelliptic. Therefore

$$
\mu \leq 4(g+1)-d_{g},
$$

where $d_{g}$ denotes the smallest degree of a curve of this type. For instance, $\mu \leq 5$ ( $\mu \leq 7$ resp.) if $g=1$ ( $g=2$ resp.). However, though (7) is sharp for $g \leq 2$, this is no longer true in general. For instance, for $g=3$ we have $d_{g}=6$, hence (7) would give $\mu \leq 10$. Nevertheless, according to [5, Theorem $4.1, \mathrm{ii})]$, the smallest degree of a rational conic fibration embedded in some projective space is 7 , hence $\mu \leq 9$.

Consider again a rational conic fibration $(X, \mathcal{L})$ where $\mathcal{L}$ is simply an ample line bundle. In view of the characterization provided by Proposition 1 we can assume that $\pi$ has $\mu \geq 1$ singular fibers. As we said, each of them consists of two (-1)-lines meeting at one point. By contracting one of these two $(-1)$-curves on every singular fiber we thus get a birational morphism $\eta: X \rightarrow X_{0}$ where $X_{0}$ is a rational $\mathbb{P}^{1}$-bundle, hence $X_{0}=\mathbb{F}_{e}$, for some $e \geq 0$. Moreover, $\mathcal{L}=\eta^{*} \mathcal{L}_{0}-\sum_{i=1}^{\mu} e_{i}$, where $e_{1}, \ldots, e_{\mu}$ are the exceptional curves contracted by $\eta$, and $\mathcal{L}_{0}$ is an ample line bundle on $\mathbb{F}_{e}$ in view of the Nakai-Moishezon criterion and the ampleness of $\mathcal{L}$. Furthermore, the fact that $2=\mathcal{L} \cdot F$ for every fiber $F$ of $X$ implies that also the fibers $f$ of $\mathbb{F}_{e}$ have degree 2 with respect to $\mathcal{L}_{0}$. So, we can write $\mathcal{L}_{0}=[2 s+\alpha f]$, for some integer $\alpha$. Note that $K_{X}=\eta^{*} K_{\mathbb{F}_{e}}+\sum_{i=1}^{\mu} e_{i}$. Since $K_{\mathbb{F}_{e}}=[-2 s-(e+2) f]$, the sectional genus $g$ of $(X, \mathcal{L})$ is given by

$2 g-2=\mathcal{L} \cdot\left(K_{X}+\mathcal{L}\right)=\mathcal{L}_{0} \cdot\left(K_{\mathbb{F}_{e}}+\mathcal{L}_{0}\right)=[2 s+\alpha f] \cdot(\alpha-2-e) f=2(\alpha-2-e)$. Thus $\mathcal{L}_{0}=[2 s+(e+g+1) f]$ and the ampleness implies $e \leq g$. However, we can say more. Consider the points $p_{i}=\eta\left(e_{i}\right), i=1, \ldots, \mu$. Clearly $p_{1}, \ldots, p_{\mu}$ lie on distinct fibers of $\mathbb{F}_{e}$. Suppose that $t$ of these points, e.g. the first $t, p_{1}, \ldots, p_{t}$, lie on the section $s$ (on the same section $s$ if $e=0$ ).

Proposition 2. Let $\eta: X \rightarrow \mathbb{F}_{e}$ be a birational morphism expressing $X$ as the blow-up of $\mathbb{F}_{e}$ at points $p_{1}, \ldots, p_{\mu}$ lying on distinct fibers, with some of them, say $p_{1}, \ldots, p_{t}$ with $t \geq 1$ belonging to the $(-e)$-section $s$. Then there exists a birational morphism $\eta^{\prime}: X \rightarrow \mathbb{F}_{\text {e+1 }}$ expressing $X$ as the blow-up of 
$\mathbb{F}_{e+1}$ at points $p_{1}^{\prime}, \ldots, p_{\mu}^{\prime}$ lying on distinct fibers, with only $t-1$ of them, say $p_{2}^{\prime}, \ldots p_{t}^{\prime}$, belonging to the $(-(e+1))$-section $s^{\prime}$ of $\mathbb{F}_{e+1}$, and conversely.

Proof. By [9, Section 2] (see also [11, Ch. V. $§ 1$, pp. 85-89]) the elementary transformation $\operatorname{elm}_{p_{1}}$ centered at $p_{1}$, gives rise to a commutative diagram

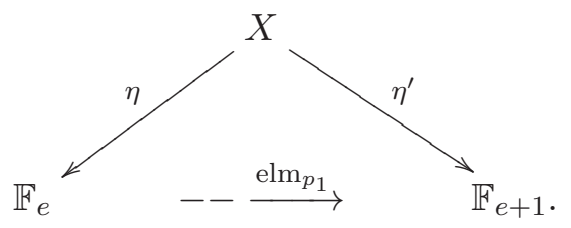

where $\eta^{\prime}: X \rightarrow \mathbb{F}_{e+1}$ is the blowing up of $\mathbb{F}_{e+1}$ at $p_{1}^{\prime}, \ldots, p_{\mu}^{\prime}$, with $p_{i}^{\prime}=$ $\operatorname{elm}_{p_{1}}\left(p_{i}\right)$ for $i=2, \ldots, \mu$ and $p_{1}^{\prime}=\operatorname{elm}_{p_{1}}\left(f_{1}\right)$, where $f_{1}$ is the fiber of $\mathbb{F}_{e}$ through $p_{1}$, since $p_{1} \in s$. Then $s^{\prime}:=\operatorname{elm}_{p_{1}}(s)$ is the $(-(e+1))$-section of $\mathbb{F}_{e+1}$ and $p_{i}^{\prime} \in s^{\prime}$ for $i=2, \ldots, t$, while $p_{1}^{\prime} \notin s^{\prime}$.

Up to an iterated application of Proposition 2, we can thus assume that $e=1$, i.e., $\eta: X \rightarrow \mathbb{F}_{1}$, with $p_{1}, \ldots, p_{\mu}$ lying on distinct fibers. As a consequence, $\mathcal{L}_{0}=[2 s+(g+2) f]$. By the way we note that

$$
\mathcal{L}^{2}=\mathcal{L}_{0}^{2}-\mu=-4 e+4(g+2)-\mu=4(g+1)-\mu,
$$

as already pointed out in (므).

The above discussion can be summed up in the following structure theorem for polarized rational conic fibrations (compare with [5, Proposition 3.1, i) for $g=2$ and Theorem 4.1, ii) for $g=3$ ] when $\mathcal{L}$ is very ample).

Theorem 3. Let $(X, \mathcal{L})$ be a rational conic fibration of sectional genus $g$. Then either

(1) $(X, \mathcal{L})=\left(\mathbb{F}_{e},[2 s+(g+e+1) f]\right)$ for some $0 \leq e \leq g$, or

(2) $X$ has $\mu>0$ singular fibers, it is obtained by blowing-up $\mathbb{F}_{1}$ at points $p_{1}, \ldots, p_{\mu}$ lying on distinct fibers, and $\mathcal{L}=\eta^{*}[2 s+(g+2) f]-\sum_{i=1}^{\mu} e_{i}$, where $\eta: X \rightarrow \mathbb{F}_{1}$ is the blow-up.

In the following, we will explore some further restrictions on the location of the $p_{i}$ 's deriving from the ampleness of $\mathcal{L}$. First of all we note the following fact.

Proposition 4. Suppose that $p_{1}, \ldots, p_{t}$ belong to the $(-1)$-section $s$ of $\mathbb{F}_{1}$. Then $t \leq g-1$.

Proof. Let $\sigma:=\eta^{-1}(s)$ be the proper transform of $s$ via $\eta$. Then $\sigma=$ $\eta^{*} s-\sum_{i=1}^{t} e_{i}$, since $s$ is smooth. Therefore its degree with respect to $\mathcal{L}$ is given by

$$
\sigma \cdot \mathcal{L}=\left(\eta^{*} s-\sum_{i=1}^{t} e_{i}\right) \cdot\left(\eta^{*} \mathcal{L}_{0}-\sum_{i=1}^{\mu} e_{i}\right)=s \cdot \mathcal{L}_{0}-t=g-t .
$$

Then the assertion follows from the ampleness of $\mathcal{L}$. 
An advantage of looking at $\eta: X \rightarrow \mathbb{F}_{1}$ is that, as we said, $\mathbb{F}_{1}$ is $\mathbb{P}^{2}$ blownup at a point, say $q_{0}$, the tautological section $s$ of $\mathbb{F}_{1}$ being the exceptional curve of the blow-up $\tau: \mathbb{F}_{1} \rightarrow \mathbb{P}^{2}$ of $\mathbb{P}^{2}$ at $q_{0}$. Set $q_{i}:=\tau\left(p_{i}\right)$ for every $i=1, \ldots, \mu$. Composing $\eta$ with $\tau$ we can look at the birational morphism $\theta:=\tau \circ \eta: X \rightarrow \mathbb{P}^{2}$, which allows us to regard $X$ as the projective plane blown-up at the $\mu+1$ points $q_{0}, q_{1}, \ldots, q_{\mu}$. Note that $q_{0}$ cannot be collinear with two other points $q_{i}$ and $q_{j}$, otherwise their preimages $p_{i}$ and $p_{j}$ via $\tau$ would belong to the same fiber of $\mathbb{F}_{1}$, which is not the case.

Moreover, given a point, say $p_{1}$, on $s$, the corresponding point $q_{1} \in \mathbb{P}^{2}$ is an infinitely near point to $q_{0}$. According to Proposition 4, we thus see that at most $g-1$ of the $q_{i}$ 's $(i=1, \ldots, \mu)$ can be infinitely near points to $q_{0}$.

More generally, if $\mu>0$, we can observe the following fact.

Remark 3. Up to renaming, suppose that $p_{1}, \ldots, p_{\nu}$ with $\nu \leq \mu$ lie on an irreducible section $s^{\prime}$ of $\mathbb{F}_{1}$. Since $s^{\prime} \in|s+\beta f|$ for some $\beta \geq 0$ (so $s^{\prime}=s$ if and only if $\beta=0)$, letting $\sigma^{\prime}=\eta^{-1}\left(s^{\prime}\right)$, the same computation as in (8) gives $\sigma^{\prime} \cdot \mathcal{L}=2 \beta+g-\nu$, and therefore, the ampleness of $\mathcal{L}$ implies that no more than $g+2 \beta-1$ of the points $p_{i}$ can lie on an irreducible section $s^{\prime} \in|s+\beta f|$ of $\mathbb{F}_{1}$. Looking at the blowing-up $\tau: \mathbb{F}_{1} \rightarrow \mathbb{P}^{2}$ and recalling what we said in Section 2, we know that for $\beta=1$ ( $\beta=2$ respect.), $s^{\prime}$ is the proper transform of a line in $\mathbb{P}^{2}$ not containing $q_{0}$ (of an irreducible conic passing through $q_{0}$ respect.). Thus, for $g=1$ the above restriction is equivalent to the requirement that no three of the $q_{i}$ 's in $\mathbb{P}^{2}(i=1, \ldots, \mu)$ are collinear and that at most four of them can lie on an irreducible conic passing through $q_{0}$. These conditions are well-known in view of the classification of del Pezzo surfaces. Similarly, for $g=2$, we have that at most three of the $q_{i}$ 's $(i=1, \ldots, \mu)$ can be collinear and at most five of them can lie on an irreducible conic passing through $q_{0}$. In the same way, looking at the proper transform $\gamma$ of a general element in $|2 s+2 f|$, i.e. the proper transform via $\theta$ of a general conic not passing through $q_{0}$, we conclude that no more than $2 g+3$ of the $q_{i}$ 's $(i=1, \ldots, \mu)$ can lie on a general conic in $\mathbb{P}^{2}$ (i.e., no more than 5 for $g=1$ and no more than 7 for $g=2$ ). Let us note the following consequence. If $g=2$, then $q_{0}, q_{1}, \ldots, q_{6}$ cannot lie on an irreducible conic, otherwise $\mathcal{L}$ could not be ample. On the other hand, $q_{1}, \ldots, q_{7}$ lying on an irreducible conic does not affect the ampleness of $\mathcal{L}$. However, this prevents $\mathcal{L}$ from being very ample (see Remark 4 in Sec. 4).

Along the same line we can investigate further restrictions on the $p_{i}$ 's in connection with any irreducible curve $C \in|2 s+\beta f|$ for $\beta \geq 3$. In this case, $p_{a}(C) \geq 1$ and, since $C \cdot f=2$, if $p_{i} \in C$, then $\operatorname{mult}_{p_{i}}(C) \leq 2$, so $C$ can have at worst double points, provided that their number does not exceed $p_{a}(C)$. For instance, letting $\beta=3$ we have $p_{a}(C)=1$, hence $C$ can have one double point at most. So, if e. g. $C$ has a double point at $p_{1}$ and passes through $p_{2}, \ldots, p_{\nu}$, with $\nu \leq \mu$, then from

$$
C \cdot \mathcal{L}=\left(\eta^{*}(2 s+3 f)-2 e_{1}-\sum_{j=2}^{\nu} e_{j}\right) \cdot\left(\eta^{*}(2 s+4 f)-\sum_{i=1}^{\mu} e_{i}\right)=9-\nu,
$$


we see that if $\mu \geq 9$, the ampleness of $\mathcal{L}$ prevents that 9 of the $p_{i}$ 's lie on a curve $C \in|2 s+3 f|$ having a double point at one of them. This translates in terms of plane curves by saying that $q_{0}, q_{1}, \ldots, q_{9}$ cannot lie on a cubic having a double point at a $q_{i}$ with $i>0$.

A relevant consequence of (5) is the ampleness or the very ampleness of $\mathcal{L}$ for $g \geq 2$, provided that the anticanonical bundle $-K_{X}$ is good enough. In fact we have

Proposition 5. Let $(X, \mathcal{L})$ be a pre-conic fibration for some line bundle $\mathcal{L}$, let $F$ be a fiber, and set $\mathcal{A}_{m}:=-K_{X}+m F$ for any positive integer $m$.

(1) If $-K_{X}$ is nef then $\mathcal{A}_{m}$ is ample for any $m$.

(2) If $-K_{X}$ is nef and big then $\mathcal{A}_{m}$ is very ample for any $m$.

Note that $\mathcal{A}_{g-1}=\mathcal{L}$, by (5) .

Proof. We have $\mathcal{A}_{m}=\mathcal{A}_{1}+(m-1) F$. So, since the line bundle corresponding to a fiber is spanned, it is enough to prove both assertions for $\mathcal{A}:=\mathcal{A}_{1}$, 4 , Ex. 7.5(d), p. 169]. If $-K_{X}$ is nef, then $\mathcal{A}$ is nef, being the sum of two nef line bundles; moreover $\mathcal{A}^{2}=K_{X}^{2}-2 K_{X} \cdot F+F^{2} \geq 4$, since $K_{X}^{2} \geq 0,-K_{X}$ being nef, and $K_{X} \cdot F=-2$ by the genus formula. So, if $\mathcal{A}$ is not ample then there exists an irreducible curve $C \subset X$ such that $0=\mathcal{A} \cdot C=-K_{X} \cdot C+F \cdot C$, in view of the Nakai-Moishezon criterion. Since both summands are nonnegative, this implies that $K_{X} \cdot C=F \cdot C=0$. The latter condition says that $C$ is contained in a union of fibers, so, due to the structure of $(X, \mathcal{L})$, it is either a fiber or an irreducible component of a singular fiber, hence a $(-1)$-curve. In both cases the equality $K_{X} \cdot C=0$ cannot hold, and therefore $\mathcal{A}$ is ample.

To prove the second assertion, write $\mathcal{A}=K_{X}+M$ where $M=-2 K_{X}+F$. Note that $M$ is nef, so being both $-K_{X}$ and $F$. Moreover, $M^{2}=4 K_{X}^{2}-$ $4 K_{X} \cdot F=4 K_{X}^{2}+8 \geq 12$, since $-K_{X}$ is also big by assumption, and $K_{X} \cdot F=-2$ as already observed. We can thus apply Reider's theorem [10, Theorem]: if $\mathcal{A}$ is not very ample, then $X$ has to contain a divisor $D>0$ such that one of the following holds:

i) $D \cdot M=0$ and $D^{2}=-2$ or -1 ;

ii) $D \cdot M=1$ and $D^{2}=-1$ or 0 ;

iii) $D \cdot M=2$ and $D^{2}=0$.

Let us prove that neither of the cases above may occur.

In case i), due to the expression of $M$, it must be $D \cdot K_{X}=D \cdot F=$ 0 . The latter condition says that $D$ is contained in a union of fibres, but this immediately leads to a contradiction: actually, since every singular fiber consists of two $(-1)$-curves meeting at one point, we can write $D=$ $\sum_{j=1}^{u} s_{j} F_{j}+\sum_{i=1}^{v} r_{i} \ell_{i}$ where the $F_{j}$ 's are fibers, the $\ell_{i}$ 's are irreducible components of singular fibers and $s_{1}, \ldots s_{u}, r_{1}, \ldots r_{v}$ are positive integers; then $D \cdot K_{X}=-2 \sum_{j=1}^{u} s_{j}-\sum_{i=1}^{v} r_{i}$ cannot be zero. 
In case ii), it must be $D \cdot K_{X}=0$ and $D \cdot F=1$. Moreover, the genus formula implies that $D^{2}=0$. Since $-K_{X}$ is big the Hodge index theorem shows that $D$ has to be numerically trivial, but this contradicts $D \cdot F=1$.

In case iii) we get either: j) $D \cdot K_{X}=0$ and $D \cdot F=2$, or jj) $D \cdot\left(-K_{X}\right)=1$ and $D \cdot F=0$. The same argument as in ii) rules out subcase j). On the other hand, subcase jj) cannot occur in view of the genus formula. This concludes the proof.

\section{Sectional genus two}

In this Section $(X, \mathcal{L})$ will be a rational conic fibration with $g=2$. First assume that $\mathcal{L}$ is simply an ample line bundle. If $\mu \geq 1$, Proposition 4 says that $t \leq 1$, i.e. at most one of the points $p_{1}, \ldots, p_{\mu}$ can lie on the tautological section $s$. Recalling the description in terms of the birational morphism $\theta$ : $X \rightarrow \mathbb{P}^{2}$ provided in Sec. 3, this can be translated into the following result, which improves [5, Remark 3.2] even in the setting of polarized surfaces.

Proposition 6. If $g=2$ then one at most of the $q_{i}$ 's can be an infinitely near point to $q_{0}$.

Furthermore, by specializing (15), we have

$$
\mathcal{L}=-K_{X}+F,
$$

where $F$ stands for a fiber of $X$. Moreover, $d=12-\mu$ by (6) . Relation (9) allows us to prove the following result, which provides a partial converse to Proposition 5 (2).

Proposition 7. Let $(X, \mathcal{L})$ be a rational conic fibration of sectional genus 2 with $\mathcal{L}$ very ample. Then $-K_{X}$ is nef and big.

Proof. If $X=\mathbb{F}_{e}$, with $e \leq 2$, then $-K_{X}$ is obviously ample for $e \leq 1$, and nef and big for $e=2$. So, according to Theorem 3 and Remark 2, we can suppose that $X$ is obtained via a blow-up $\eta: X \rightarrow \mathbb{F}_{1}$ at $1 \leq \mu \leq 7$ points lying on distinct fibers, one at most, say $p_{1}$, belonging to the minimal section $s$ of $\mathbb{F}_{1}$, and the line bundle $\mathcal{L}$ is given by $\mathcal{L}=\eta^{*}[2 s+4 f]-\sum_{i=1}^{\mu} e_{i}$. Recalling (2), we get $h^{0}(2 s+4 f)=12$. Thus $h^{0}(\mathcal{L}) \geq h^{0}(2 s+4 f)-\mu \geq 5$, because $\mu \leq 7$. Since $\mathcal{L} \cdot F=2$, we thus get $\operatorname{dim}(|\mathcal{L}-F|)=\operatorname{dim}(|\mathcal{L}|)-3 \geq 1$ and then, due to (9), $\left|-K_{X}\right|=|\mathcal{L}-F|$ is at least a pencil. Clearly, $K_{X}^{2}=K_{\mathbb{P}^{2}}^{2}-\mu-1=8-\mu \geq 1$, hence $-K_{X}$ is big provided that it is nef. Suppose, that $-K_{X}$ is not nef. Then there exists an irreducible curve $C \subset X$ such that $C \cdot(\mathcal{L}-F)=C \cdot\left(-K_{X}\right)<0$, i.e.,

$$
C \cdot \mathcal{L}<C \cdot F \text {. }
$$

As $C \cdot D<0$ for every $D \in\left|-K_{X}\right|=|\mathcal{L}-F|$, necessarily the curve $C$ has to be contained in the fixed part of $|\mathcal{L}-F|$, so we can write $|\mathcal{L}-F|=C+|R|$, where $R$ is an effective divisor. Thus

$$
2=\mathcal{L} \cdot F=(\mathcal{L}-F) \cdot F=C \cdot F+R \cdot F \geq C \cdot F,
$$


because $F$ is nef. On the other hand, the ampleness of $\mathcal{L}$ implies $C \cdot \mathcal{L} \geq 1$. Combining this with the above inequalities we get

$$
1 \leq C \cdot \mathcal{L}<C \cdot F \leq 2
$$

and this in turn implies

$$
C \cdot \mathcal{L}=1 \quad \text { and } \quad C \cdot F=2 .
$$

We claim that this exceptional situation cannot happen $\mathcal{L}$ being very ample. Suppose (10) holds. Then $C$ is a line on $X \subset \mathbb{P}^{N}$ embedded by $|\mathcal{L}| ;$ moreover, $C$ is contained in the linear span $\langle F\rangle \subset \mathbb{P}^{N}$ of every fiber $F$, since it intersects every fiber at two points. As a consequence, the planes $\langle F\rangle$ constitute a pencil whose axis is the line $C$ itself, and so $X$ is contained in the $\mathbb{P}^{3}$ spanned by that pencil. But the only smooth surface in $\mathbb{P}^{3}$ admitting a structure of conic fibration is the cubic surface, and this is not compatible with $g=2$. Therefore (10) cannot occur.

More generally, we have

Proposition 8. Let $(X, \mathcal{L})$ be a rational conic fibration of sectional genus 2 and suppose that $\mathcal{L}$ is ample and spanned. Then either

a) $8 \leq \mu \leq 9$, or

b) $-K_{X}$ is nef and big, unless

(11) $\mu=7, t=0$, and $p_{1}, \ldots, p_{7}$ belong to an irreducible curve of $|2 s+2 f|$.

Proof. By Remark 2 we know that $\mu \leq 9$ since $\mathcal{L}$ is ample and spanned. Suppose we are not in case a). Then $\mu \leq 7$ and so we can argue as in the part of the proof of Proposition 7 leading to formula (10) under the assumption that $-K_{X}$ is not nef. Then, $C$ is a bisecant of the fibration. Moreover, the fact that $\mathcal{L}_{C}$ is an ample and spanned line bundle of degree 1 on $C$ implies that $C$ is a smooth rational curve. Consider the birational morphism $\eta: X \rightarrow \mathbb{F}_{1}$ again and set $\gamma:=\eta(C)$; then $\gamma \subset \mathbb{F}_{1}$ is an irreducible curve. We can write $\gamma \sim a s+b f$ for some integers $a$ and $b$, and $C=\eta^{*} \gamma-\sum_{i=1}^{\mu} \nu_{i} e_{i}$, for suitable non-negative integers $\nu_{i}=\operatorname{mult}_{p_{i}}(\gamma)$. As $F=\eta^{*} f$, we have

$$
2=C \cdot F=\left(\eta^{*} \gamma-\sum_{i=1}^{\mu} \nu_{i} e_{i}\right) \cdot \eta^{*} f=\gamma \cdot f=a,
$$

and this implies $0 \leq \nu_{i} \leq 2$ for every $i=1, \ldots, \mu$. So, if $\gamma$ is singular, it can have at worst double points at some of the $p_{i}$ 's and since $C$ is rational, their number is exactly $p_{a}(\gamma)$. By the genus formula we have

$$
2 p_{a}(\gamma)-2=\gamma \cdot\left(K_{\mathbb{F}_{1}}+\gamma\right)=(2 s+b f) \cdot(b-3) f=2(b-3),
$$

hence $p_{a}(\gamma)=b-2$. Let $\varepsilon$ be the number of the $p_{i}$ 's for which $\nu_{i}=1$. Then $b-2+\varepsilon \leq \mu \leq 7$. Thus, we get

$$
1=C \cdot \mathcal{L}=\left(\eta^{*}[2 s+b f]-\sum_{i=1}^{\mu} \nu_{i} e_{i}\right) \cdot\left(\eta^{*}[2 s+4 f]-\sum_{i=1}^{\mu} e_{i}\right)
$$




$$
=-4+8+2 b-2(b-2)-\varepsilon=8-\varepsilon .
$$

Therefore $7=\varepsilon=\mu$ and $b-2=0$. So, $\gamma$ is a smooth curve of $|2 s+2 f|$ containing all the seven $p_{i}$ 's. In particular, $t=0$ in view of Remark 1 , and then assertion b) in the statement is proved.

Remark 4. The special case (11) arising in Proposition 8 is an effective exception to the nefness of $-K_{X}$. To see this, referring to the blow-up $\tau: \mathbb{F}_{1} \rightarrow \mathbb{P}^{2}$ and to $\theta=\tau \circ \eta: X \rightarrow \mathbb{P}^{2}$, consider that $[2 s+2 f]=\tau^{*} \mathcal{O}_{\mathbb{P}^{2}}(2)$. Then the exceptional situation in (11) corresponds to the fact that the seven points $q_{i}=\tau\left(p_{i}\right)(i=1, \ldots, 7)$ of $\mathbb{P}^{2}$ lie on an irreducible conic. According to [2, Théorème 1, p. 39], this fact prevents $-K_{X}$ from being nef (and hence $\mathcal{L}$ from being very ample).

Here is an easy application of (9) and Proposition 7 . Clearly, in case $t=1$, the proper transform of the tautological section $s$ via $\eta: X \rightarrow \mathbb{F}_{1}$ is a $(-2)$-curve which is a line of $(X, \mathcal{L})$ and a section of $X$. In fact we can say more.

Proposition 9. Let $X \subset \mathbb{P}^{N}$ be a rational conic fibration of sectional genus 2 with $\mu>0$, let $\eta: X \rightarrow \mathbb{F}_{1}$ be the morphism expressing $X$ as the blow-up of $\mathbb{F}_{1}$ at $p_{1}, \ldots, p_{\mu}$, and let $C \subset X$ be a line. Then, either

1) $C$ is a component of a singular fiber of $X$, or

2) $C$ is a (-2)-curve and a section of $X$.

Proof. Let $\mathcal{L}$ be the hyperplane bundle of $X$ and let $C \subset X$ be a line of $(X, \mathcal{L})$. In view of the relation (9) we have $1=C \cdot \mathcal{L}=C \cdot\left(-K_{X}\right)+C \cdot F$. Clearly, $F$ is nef. Moreover, $-K_{X}$ is also nef in view of Proposition 7 , then either

i) $C \cdot K_{X}=-1$ and $C \cdot F=0$, or

ii) $C \cdot K_{X}=0$ and $C \cdot F=1$,

and in these two cases $C$ is as in 1) and 2) respectively.

\section{Characterizing the very ampleness of $\mathcal{L}$ for $g=2$}

Notation as in Section 3. Let $\eta: X \rightarrow \mathbb{F}_{1}$ be the blowing up of $\mathbb{F}_{1}$ at points $p_{1}, \ldots, p_{\mu}$ with $\mu \leq 11$, lying on distinct fibers, and let $\mathcal{L}=$ $\eta^{*}[2 s+4 f]-\sum_{i=1}^{\mu} e_{i}$. Since $\bar{g}([2 s+4 f])=2>0$, if $p_{1}, \ldots, p_{\mu}$ are general, then $\mathcal{L}$ is ample, according to Yokoyama [12, Theorem, 1.8]. Then $(X, \mathcal{L})$ is a polarized rational conic fibration of sectional genus 2 . On the other hand, if $p_{1}, \ldots, p_{\mu}$ are not general, the "bad curves" containing some of them and preventing $\mathcal{L}$ from being ample include those mentioned in Section 3 , but there could be more. In this Section, as a consequence of Proposition 5 and Proposition 7 we succeed to describe precisely the conditions on the location of $p_{1}, \ldots, p_{\mu}$ characterizing the very ampleness of $\mathcal{L}$ for $\mu \leq 7$. To do that we need to translate the condition that $q_{0}, q_{1}, \ldots, q_{\mu}$ are in almost general position according to [2, p. 39] in terms of the points $p_{1}, \ldots, p_{\mu}$ on $\mathbb{F}_{1}$. The relevant fact is that, while the same conditions are obviously sufficient for 
the mere ampleness of $\mathcal{L}$, they turn out also to be necessary except in the geometric situation specified by (11).

So, let $(X, \mathcal{L})$ be a rational conic fibration of sectional genus $g=2$ and consider the blow-up $\theta: X \rightarrow \mathbb{P}^{2}$ at $q_{0}, q_{1}, \ldots, q_{\mu}$ where $\mu \leq 7$ (we can assume this fact since it is a necessary condition for the very ampleness of $\mathcal{L}$; see Remark 2). According to [2, Théorème 1, equivalence of conditions (a) and (d), p. 39], $-K_{X}$ is nef if and only if $q_{0}, q_{1}, \ldots, q_{\mu}$ are in almost general position (see [2, Definition 1, p. 39]). Note that property $(*)$ in [2, point b) p. 38] is certainly satisfied in the present case in view of Proposition 6. This means that the first requirement in [2, Definition 1, p. 39] is fulfilled, and therefore the points $q_{0}, q_{1}, \ldots, q_{\mu}$ are in almost general position if and only if they are

i) no four on a line, and

ii) no seven on a conic.

Before translating these conditions in terms of the points $p_{1}, \ldots, p_{\mu}$ on $\mathbb{F}_{1}$, we note that if $t=1$ (i.e. $q_{1}$ is infinitely near to $q_{0}$ ), then a line or a conic through $q_{1}$ has to contain $q_{0}$.

So, let us translate the complementary situation of i).

i-1) suppose that $q_{0}, q_{i_{1}}, q_{i_{2}}, q_{i_{3}}$ lie on a line. This means that the three points $p_{i_{1}}, p_{i_{2}}, p_{i_{3}}$ belong to the same fiber of $\mathbb{F}_{1}$ (which is not the case since $\mathcal{L}$ is ample);

i-2) suppose that $q_{i_{1}}, q_{i_{2}}, q_{i_{3}}, q_{i_{4}}$ are on a line, the 4-tuple not including $q_{0}$ (and then not even $q_{1}$ if $t=1$ ). This means that the four points $p_{i_{1}}, p_{i_{2}}, p_{i_{3}}, p_{i_{4}}$ belong to an irreducible curve in the linear system $|s+f|$ (but this contradicts the ampleness of $\mathcal{L}$ : immediate check).

Therefore no four of the $p_{i}$ 's can lie on an irreducible curve in $|s+f|$. However, three can lie on such a curve if either $t=0$, or $t=1$ provided that $p_{1}$ is not one of them (recalling Remark 1 ).

Next let us translate the complementary situation of ii). Of course this requires $\mu=6$ or 7 .

ii-1) suppose that the conic contains $q_{0}$. This means that six of the points $p_{i}$ belong to an irreducible curve in the linear system $|s+2 f|$ (an immediate check shows that this contradicts the ampleness of $\mathcal{L}$ );

ii-2) suppose that the conic misses $q_{0}$ (and then, necessarily, $t=0$ ); this means that the seven points $p_{1}, \ldots, p_{7}$ belong to an irreducible curve in the linear system $|2 s+2 f|$ (this is the special case (11)) in Proposition 8 b), which does not contradict the ampleness of $\mathcal{L}$ ).

Here is the main result.

Theorem 10. Let $(X, \mathcal{L})$ be a rational conic fibration of sectional genus 2 with $\mathcal{L}$ ample and $\mu \leq 7$ singular fibers.

j) Then $(X, \mathcal{L})$ is as in point (2) of Theorem 3, with $g=2$; moreover, the points $p_{1}, \ldots, p_{\mu}$ satisfy the following conditions: at most one can lie on s; no four are on an irreducible curve in the linear system 
$|s+f|$, and no six are on an irreducible curve in $|s+2 f|$. Conversely, if these conditions are satisfied for a pre-conic fibration $(X, \mathcal{L})$ with $g=2$, then $\mathcal{L}$ is ample.

jj) Moreover, if all the above conditions are satisfied, then $\mathcal{L}$ is spanned.

jjj) Furthermore, $\mathcal{L}$ is very ample if and only if the above conditions are satisfied and, in addition, in case $\mu=7, t=0$ and the points $p_{1}, \ldots, p_{\mu}$ do not belong to an irreducible curve in $|2 s+2 f|$.

Proof. j) follows from the above discussion, taking also into account Proposition 4. Assertions jj) and jjj) are trivial if $\mu=0$. So let $\mu \geq 1$ and consider the blow-up $\eta: X \rightarrow \mathbb{F}_{1}$ again. Since $\mathcal{L}=\eta^{*}[2 s+4 f]-\sum_{i=1}^{\mu} e_{i}$, we see from [1, Theorem 4.1] that the sufficient conditions for the spannedness of $\mathcal{L}$ are weaker than those listed in j); this implies jj). Finally, jjj) follows from Proposition 5(2) and Proposition 7, taking into account jj) and Proposition 8.

Note that the conditions in $\mathrm{j}$ ) are also sufficient for the ampleness of $\mathcal{L}$ when $\mu \leq 6$. Actually, the ampleness of $\mathcal{L}$ follows from Proposition 5 (1) and [2, Théorème 1], since the conditions in j) express the nefness of $-K_{X}$ for $\mu \leq 6$. On the other hand, if $\mu=7$, then either $-K_{X}$ is nef and then $\mathcal{L}$ is ample, or $(X, \mathcal{L})$ is as in (11) (which, however does not prevent $\mathcal{L}$ from being ample.

Corollary 11. Let $(X, \mathcal{L})$ be a polarized rational conic fibration with $g=2$. If $\mu \leq 7$ then $\mathcal{L}$ is very ample, except if (11) holds.

Remark 5. We used Bese's paper [1 for the spannedness of $\mathcal{L}$ but we did not for the very ampleness. Actually, according to [1, Theorem 4.2], $\mathcal{L}$ would be very ample provided that for any curve $C \in|x s+y f|$ with $x$ and $y$ nonnegative integers no both zero, such that $x \leq 4, y \leq 3$,

at most $\nu:=x(x-y+3)+y(4-x)-3$ of the $p_{i}$ 's belong to $C$.

Let $\mu=7$. Note that $\nu>7$ for all admissible pairs $(x, y)$ with $x \geq 3$, hence requirement (12) is meaningful only for the restricted subset of admissible $(x, y)$ with $x \leq 2$. Moreover, (12) is obvious when $x=0$ because the seven points lie on distinct fibers; similarly, (12) is obvious also when $y=0$, since in this case $C=s$ or $2 s$ according to whether $x=1$ or 2 , and we know that at most one of the $p_{i}$ 's can lie on $s$. This reduces the analysis to $11-5=6$ admissible pairs and a close check can be done. In particular, recalling that $\mu=7$, in addition to the conditions in $\mathrm{j}$ ) of Theorem 10, we see that (12) includes some further restrictions, like that the seven point cannot lie on a curve in $|s+3 f|$ or in $|2 s+3 f|$. Looking at the plane model and using the usual notation, we see that these curves are the proper transforms on $\mathbb{F}_{1}$ of a cubic passing through $q_{0}$ with multiplicity 2 or 1 respectively. However, the fact that these curves do not enter at all in our conditions (see jjj) of Theorem 10) is not a trouble once we consider that Bese's conditions are only sufficient conditions (but not necessary) for the very ampleness of $\mathcal{L}$. 
For the remaining $(x, y)$, (12) is satisfied in view of the conditions in $\mathrm{j}$ ) of Theorem 10. The key point, however, is that this happens also if the seven points lie on an irreducible curve $C \in|2 s+2 f|$. Thus $\mathcal{L}$ would be very ample even in this case according to Bese, but we stress that this is in contrast with what we proved (this is exactly the exceptional configuration in (11)).

\section{RAtional CONIC FibRATIONS With $g=2$ CONTAining LinES}

Finally, in this Section we characterize the rational conic fibrations $(X, \mathcal{L})$ of sectional genus 2 , with $\mathcal{L}$ very ample, containing a line $C$ transverse to the fibers.

If $X=\mathbb{F}_{e}$ with $e \leq 2$, then $\mathcal{L}=[2 s+(e+3) f]$ by Theorem 3 . Since $C$ is an irreducible curve we have either $C=s$ or $C \sim a s+b f$ for some integers $a$ and $b$ such that $b \geq a e$. Moreover, $a=1$ or 2 because $C$ is transverse to the fibers and $C \cdot f \leq 2$ since $C$ is a line and $f$ is a conic. In the former case, from $1=C \cdot \mathcal{L}=s \cdot[2 s+(e+3) f]=3-e$ we get $e=2$. In the latter, we get $1=C \cdot \mathcal{L}=(a s+b f) \cdot[2 s+(e+3) f]=-2 a e+2 b+a(e+3) \geq a(e+3) \geq 3$, a contradiction. Therefore $e=2$ and $C=s$. On the other hand, as $s \cdot(2 s+5 f)=1$ we have that this curve is in fact a line of $(X, \mathcal{L})$. Next, suppose that $\mu \geq 1$ and use the description via the birational morphism $\eta$, recalling that $\mu \leq 7$ since $\mathcal{L}$ is very ample. By Proposition 9 we know that $C$ is a section of $X$ with $C^{2}=-2$. Then $\gamma:=\eta(C)$ is a section of $\mathbb{F}_{1}$, hence $\gamma \in|s+\alpha f|$ for some integer $\alpha \geq 0$. Moreover, $C=\eta^{*} \gamma-\sum_{i=1}^{\mu} \nu_{i} e_{i}$, where $\nu_{i}=\operatorname{mult}_{p_{i}}(\gamma)=C \cdot e_{i} \leq 1$ for every $i=1, \ldots, \mu$, because both $C$ and $e_{i}$ are lines in $X$ embedded in some $\mathbb{P}^{N}$ by $|\mathcal{L}|$. Let $\varepsilon$ be the number of the $p_{i}$ 's for which $\nu_{i}=1$. Then

$$
-2=C^{2}=\gamma^{2}-\varepsilon=2 \alpha-1-\varepsilon .
$$

Thus $\alpha=\frac{1}{2}(\varepsilon-1)$, so that $\varepsilon$ has to be odd. If $\varepsilon=1$ then $\alpha=0$, hence $\gamma=s$ and, up to renaming, $p_{1} \in s$. If $\varepsilon>1$, then, $\varepsilon=2 \alpha+1 \geq 3$. On the other hand, $\varepsilon \leq \mu \leq 7$, hence $\alpha \leq 3$. Therefore $\varepsilon=3,5$ or 7 according to whether $\alpha=1,2$ or 3 , respectively. Let $\alpha=1$; then $\gamma \in|s+f|$ and it contains three of the $p_{i}$ 's (clearly, no one of them can lie on $s$, by Remark $1)$. Next let $\alpha=2$; then $\gamma \in|s+2 f|$ and it contains five of the $p_{i}$ 's. Clearly, here $\mu \geq 5$ and it can happen that $p_{1} \in s$, because $s \cdot \gamma=s \cdot(s+2 f)=1$. Finally, if $\alpha=3$, then $\varepsilon=7=\mu$, i.e. $\gamma \in|s+3 f|$ and it contains all seven points $p_{i}$ 's. The above discussion proves the following result.

Theorem 12. Let $(X, \mathcal{L})$ be a rational conic fibration of sectional genus 2 , with $\mathcal{L}$ very ample. Then $(X, \mathcal{L})$ contains a line $C$ transverse to the fibers if and only if either

$$
(X, \mathcal{L}, C)=\left(\mathbb{F}_{2},[2 s+5 f], s\right),
$$

or $(X, \mathcal{L})$ is as in point (2) of Theorem 3 with $\mu \leq 7$ and $C=\eta^{-1}(\gamma)$, where $\gamma \subset \mathbb{F}_{1}$ is an irreducible section and one of the following conditions is satisfied:

a) $\gamma=s$ and it contains $p_{1}$; 
b) $\gamma \in|s+f|$ and it contains three of the $p_{i}$ 's;

c) $\gamma \in|s+2 f|$ and it contains five of the $p_{i}$ 's;

d) $\gamma \in|s+3 f|$ and it contains $p_{1}, \ldots, p_{7}$.

At first glance the statement of Theorem 12 does not seem to fit with [6, Proposition 8 (2)]. But there is a good reason for that since the morphism $\eta: X \rightarrow \mathbb{F}_{1}$ in point (2) of Theorem 3 we refer to here is not the same as the birational morphism $X \rightarrow \mathbb{F}_{e}$ constructed in [6]. In fact the above curve $\gamma$ has nothing to do with the curve denoted by $\ell_{0}$ in [6].

Remark 6. i) Consider the four cases occurring in Theorem 12 for $\mu \geq 1$. Clearly, in case a) we have $t=1$. In case b), let $p_{i_{1}}, p_{i_{2}}, p_{i_{3}} \in \gamma$. Referring to the blow-up $\tau: \mathbb{F}_{1} \rightarrow \mathbb{P}^{2}$ we have that $\gamma=\tau^{-1}(\ell)$, where $\ell \subset \mathbb{P}^{2}$ is a line containing three of the $q_{i}$ 's. So three of the $q_{i}$ 's are collinear. Similarly, in case c), let $p_{i_{1}}, \ldots, p_{i_{5}} \in \gamma$. Referring to $\tau$ again we have that $\gamma=\tau^{-1}(G)$, where $G \in\left|\mathcal{O}_{\mathbb{P}^{2}}(2)-q_{0}-\sum_{j=1}^{5} q_{i_{j}}\right|$. So $q_{0}$ and five more points $q_{i}$ 's (possibly one of them being infinitely near to $q_{0}$ ) are on a conic. As a consequence, in all these cases, even if $t=0$, the above conditions prevent $(X, \mathcal{L})$ from being a Castelnuovo surface, according to the definition in [6, p. 2857]. On the other hand, in case d), we have $\gamma=\tau^{-1}(\Gamma)$, where $\Gamma \in \mid \mathcal{O}_{\mathbb{P}^{2}}(3)-2 q_{0}-$ $\sum_{i=1}^{7} q_{i} \mid$. So the eight points $q_{j}$ 's lie on a cubic having a double point at $q_{0}$. In this case $(X, \mathcal{L})$ is in fact a Castelnuovo surface according to [6], provided that $t=0$.

ii) Looking over the proof of Theorem 12, suppose that $\gamma \neq s$ and let $\sigma$ be the proper transform of $s$; then $\sigma \neq C$. Recall that, up to renaming, $p_{1}$ at most among the $p_{i}$ 's can belong to $s$, by Proposition 6. Then $\sigma=\eta^{*} s-x e_{1}$, where $x=1$ or 0 according to whether $p_{1} \in s$ or not (i.e., $t=1$ or 0 ). We thus get $\sigma \cdot \mathcal{L}=s \cdot(2 s+4 f)-x=2-x$. Hence $\sigma$ is either a line or a conic, accordingly. In particular, $\sigma$ is a conic if and only if $t=0$. As a consequence, $\sigma \cdot C \leq 2$, since $C$ is a line. Moreover, equality means that the line $C$ lies in the plane $\langle\sigma\rangle$ spanned by $\sigma$ in the $\mathbb{P}^{N}$ where $|\mathcal{L}|$ embeds $X$. An easy computation shows that

$$
\sigma \cdot C=\left(\eta^{*} s-x e_{1}\right) \cdot\left(\eta^{*}[s+\alpha f]-\sum_{j=1}^{\varepsilon} e_{i_{j}}\right)= \begin{cases}\alpha-2 & \text { if } p_{1} \in s \cap \gamma \\ \alpha-1 & \text { otherwise. }\end{cases}
$$

This is 2 if and only if $\alpha=3$, namely in case d) of Theorem 12. Moreover, since in this case $2=\sigma \cdot C=s \cdot(s+3 f)-x=2-x$, implies $x=0$, we have $t=0$. Therefore $C \subset\langle\sigma\rangle$ if and only if $X$ embedded by $|\mathcal{L}|$ is a Castelnuovo surface of degree 5 in $\mathbb{P}^{4}$.

Now we connect the previous discussions with the inflection points of $X \subset \mathbb{P}^{N}$, linearly normally embedded by $|\mathcal{L}|$. Let us recall some general facts from [6, Section 2] in the specific case we are dealing with. Let $\mathcal{P}_{X}(\mathcal{L})$ be the second principal part bundle of $\mathcal{L}$ and let

$$
j: H^{0}(X, \mathcal{L}) \otimes \mathcal{O}_{X} \rightarrow \mathcal{P}_{X}(\mathcal{L})
$$


be the sheaf homomorphism associating to every section $\sigma \in H^{0}(X, \mathcal{L})$ its second jet $j_{x}(\sigma)$ evaluated at $x$, for every $x \in X$. Let $r:=\max _{x \in X}\left\{\operatorname{rk}\left(j_{x}\right)\right\}$ be the maximum rank of $j_{x}$ on $X$. Clearly, $3 \leq r \leq \min \{N+1,6\}$ since $\mathcal{P}_{X}(\mathcal{L})$ is a vector bundle of rank $6, X$ being a surface. A point $x \in X$ is said to an inflection point if $\operatorname{rk}\left(j_{x}\right)<r$. This is in accordance with the fact that the osculating space to $X$ at $x$ is defined as $\operatorname{Osc}_{x}(X):=\mathbb{P}\left(\operatorname{Im} j_{x}\right)$. Then the inflectional locus $\Phi(X)$ of $X$ is defined as the set of the inflection points. In view of the definition of the osculating space, the osculating hyperplanes to $X$ at $x$ can be regarded as the elements of the linear system $|\mathcal{L}-3 x|$ (hyperplane sections of $X$ having a singular point of multiplicity $\geq 3$ at $x$ ). As a consequence, $\operatorname{dim}(|\mathcal{L}-3 x|)+\operatorname{dim}\left(\operatorname{Osc}_{x}(X)\right)=N-1$, or equivalently $\operatorname{rk}\left(j_{x}\right)=N-\operatorname{dim}(|\mathcal{L}-3 x|)$. In particular, we see that

$$
\text { if }|\mathcal{L}-3 x|=\emptyset \text {, then } x \notin \Phi(X) \text {. }
$$

Coming back to the situation in Theorem [12, note that

$$
N=h^{0}(\mathcal{L})-1 \geq h^{0}(2 s+4 f)-1-\mu=11-\mu
$$

due to (2). Moreover, $N \geq 4$ since $\mathcal{L}$ is very ample. If $N \geq 5$, then the line $C$ is always contained in $\Phi(X)$, according to [6, Theorem 5 (2)]. If $N=4$, which implies $\mu=7$, we have to analyze all possible cases a) $-\mathrm{d}$ ) in Theorem 12 to decide whether the line $C$ is contained or not in $\Phi(X)$. We can argue as follows. Fix $x \in C$ and let $F_{x}$ be the fiber of $X$ passing through $x$. Since $C$ and $F_{x}$ are a line and a conic containing $x$, they are fixed components of $|\mathcal{L}-3 x|[$, , Lemma 2(1)]. Thus,

$$
\operatorname{dim}(|\mathcal{L}-3 x|)=\operatorname{dim}\left(\left|\mathcal{L}-F_{x}-C-x\right|\right)=\operatorname{dim}\left(\left|-K_{X}-C-x\right|\right),
$$

recalling (9). Keeping the notation used in the proof of Theorem 12, we can write $C \sim \eta^{*}(s+\alpha f)-\sum_{j=1}^{\varepsilon} e_{i_{j}}$, where $\varepsilon=2 \alpha+1$, with $\alpha=0,1,2,3$ according to cases a), b), c) and d) respectively in Theorem 12, Letting $P:=\left\{p_{1}, \ldots, p_{\mu}\right\}$, we thus see that $P \backslash \gamma$ consists of $\mu-\varepsilon=\mu-1-2 \alpha$ points. Thus

$$
\begin{aligned}
& \operatorname{dim}\left(\left|-K_{X}-C\right|\right)=\operatorname{dim}\left(\left|\eta^{*}(s+(3-\alpha) f)-\sum_{k=1}^{\mu-\varepsilon} e_{i_{k}}\right|\right) \\
& \geq \operatorname{dim}(|s+(3-\alpha) f|)-(\mu-1-2 \alpha),
\end{aligned}
$$

equality holding if and only if

(14) $P \backslash \gamma$ imposes linearly independent linear conditions on $|s+(3-\alpha) f|$.

Note that this certainly happens in case d) of Theorem 12, because $P \backslash \gamma$ is empty. Now, a straightforward computation shows that $h^{0}(s+(3-\alpha) f)=$ $7-2 \alpha$, hence $\operatorname{dim}\left(\left|-K_{X}-C\right|\right)=0$, provided that (14) holds and $\mu=7$. This in turn implies that

$$
\left|-K_{X}-C-x\right|=\emptyset
$$

unless $x \in D \cap C$, where $D$ is the only divisor in $\left|-K_{X}-C\right|$. But $D$ is a section and $D \cdot C=2$, hence (15) holds for the general point $x \in C$. Then, as 
a consequence of (13), $C$ is not contained in $\Phi(X)$. So we have the following result.

Proposition 13. Let $(X, \mathcal{L})$ and $C$ be as in Theorem 12 and suppose that $N=4$ (hence $\mu=7$ ). If (14) holds, then the line $C$ is not contained in the inflectional locus of $X$. In particular this applies to case $\mathrm{d})$.

Remark 7. In fact, as to case d) we proved in [6. Theorem 18] that when $X \subset \mathbb{P}^{4}$ is a Castelnuovo surface, then the inflectional locus is finite and consists of 75 points, in general. We use this opportunity to point out that unlike we said in [6, Example b), p. 2870] Castelnuovo surfaces, as defined in [6, p. 2857] do not exhaust the class of surfaces of sectional genus 2 in $\mathbb{P}^{4}$. Actually, there exist surfaces with $t=0$ that are not Castelnuovo surfaces as well as examples of surfaces with $t=1$.

The following example offers an interesting situation in connection with Proposition 9, Theorem 12 and Proposition 13 ,

Example. Let $X$ be the surface obtained by blowing-up $\mathbb{F}_{1}$ at 7 points $p_{1}, \ldots, p_{7}$ lying on an irreducible curve $\gamma \in|s+3 f|$ and sufficiently general, with $p_{1} \in s$, and let $\mathcal{L}=\eta^{*}[2 s+4 f]-\sum_{i=1}^{7} e_{i}$, with obvious meaning of the symbols. Then $\mathcal{L}$ is very ample, $|\mathcal{L}|$ embeds $X$ in $\mathbb{P}^{4}$ as a quintic surface and $(X, \mathcal{L})$ is a rational conic fibration with $g=2$ and $t=1$. In particular, it is not a Castelnuovo surface in the sense of [6, p. 2857]. Let $\widetilde{s}$ and $C$ be the proper transforms on $X$ of $s$ and of $\gamma$, respectively. Both curves are lines transverse to the fibers of $X$, and in fact they are $(-2)$-curves. They correspond to case a) and to case d) respectively in Theorem 12. Since $\eta^{*} s=\widetilde{s}+e_{1}$ and $\eta^{*} \gamma=C+\sum_{i=1}^{7} e_{i}$, one immediately sees that $\widetilde{s}$ and $C$ intersect at one point, say $y_{1}$. Similarly, the line $e_{1}$, which is a component of a singular fiber, intersects both $C$ and $\widetilde{s}$. Set $y_{2}:=e_{1} \cap C$ and $y_{3}:=$

$e_{1} \cap \widetilde{s}$. Following the argument used to prove Proposition [13, we see that $-K_{X}-C=\eta^{*} s=\widetilde{s}+e_{1}$. Similarly, $-K_{X}-\widetilde{s}=\eta^{*}[s+3 f]-\sum_{i=2}^{7} e_{i}=C+e_{1}$. According to Proposition 13 the conclusion is that no $x \in C \cup \widetilde{s}$ can be an inflection point of $X$ except the three points $y_{j}, j=1,2,3$, which indeed are such.

Acknowledgements: The authors are grateful to the referee for useful remarks. The first author is a member of G.N.S.A.G.A. of the Italian INdAM. He would like to thank the PRIN 2015 Geometry of Algebraic Varieties and the University of Milano for partial support. The second author wants to thank the Spanish Ministry of Science, Innovation and Universities (Project MTM 2015-65968-P "Geometría algebraica y analítica y aplicaciones").

\section{REFERENCES}

[1] E. Bese, On the spannedness and the very ampleness of certain line bundles on the blow-ups of $\mathbb{P}_{\mathbb{C}}^{2}$ and $\mathbb{F}_{r}$, Math. Ann. 262 (1983), 225-238. 
[2] M. Demazure, Surfaces de del Pezzo, II-V, Séminaire sur les singularités des surfaces, Lect. Notes in Math., vol. 777, Springer-Verlag, Berlin, 1980.

[3] T. Fujita, Classification Theories of Polarized Varieties, London Math. Soc. Lectures Notes Ser., vol. 155, Cambridge Univ. Press, 1990.

[4] R. Hartshorne, Algebraic Geometry, Springer-Verlag, New York-HeidelbergBerlin 1977.

[5] P. Ionescu, Embedded projective varieties of small invariants, in "Proceedings of the 1982 Week of Algebraic Geometry, Bucharest", Lecture Notes in Math. 1056, Springer-Verlag 1984, pp. 142-187.

[6] A. Lanteri and R. Mallavibarrena, Osculation for conic fibrations, J. Pure Appl. Algebra 220 (2016), 2852-2878.

[7] A. Lanteri, R. Mallavibarrena, and R. Piene, Inflectional loci of quadric fibrations, J. Algebra 441 (2015), 363-397; Corrigendum, ibidem, 508 (2018), 589-591.

[8] A. Lanteri and M. Palleschi, About the adjunction process for polarized algebraic surfaces, J. reine angew. Math. 352 (1984), 15-239.

[9] M. Nagata, On rational surfaces. I. Irreducible curves of arithmetic genus 0 or 1 , Mem. Coll. Sci. Univ. Kyoto Ser. A Math. 32 (1960), 351-370.

[10] I. Reider, Vector bundles of rank 2 and linear systems on algebraic surfaces, Ann. of Math. 127 (1988), 309-316.

[11] I. R. Shafarevich et al., Algebraic Surfaces, Proc. Steklov Inst. Math. 75 (1965) (transl. Amer. Math. Soc.)

[12] K. Yokoyama, On blowing-up of polarized surfaces, J. Math. Soc. Japan 51 (1999), $523-533$.

Dipartimento di Matematica "F. Enriques", Università degli Studi di Milano, Via C. Saldini, 50, I-20133 Milano, Italy

E-mail address: antonio.lanteri@unimi.it

Departamento de Álgebra, Geometría y Topología, Facultad de Ciencias Matemáticas, Plaza de las Ciencias, 3 - Universidad Complutense de Madrid, 28040 MADRID, SPAIN

E-mail address: rmallavi@mat.ucm.es 